\title{
Decision Rules in Cost-Utility Analysis of Health Technologies
}

\section{Udomsak SAENGOW ${ }^{1,2}$}

\author{
${ }^{1}$ Center of Excellence in Health System and Medical Research, Walailak University, \\ Nakhon Si Thammarat 80160, Thailand \\ ${ }^{2}$ School of Medicine, Walailak University, Nakhon Si Thammarat 80160, Thailand
}

\author{
(Corresponding author’s e-mail: saengow.udomsak@gmail.com)
}

Received: 21 August 2019, Revised: 18 February 2020, Accepted: 28 March 2020

\begin{abstract}
This review identified 4 distinct decision rules used in the cost-utility analysis of health technologies: threshold incremental cost-effectiveness ratio (ICER), league table, decision making (DM) plane, and linear programming. The threshold ICER is currently the most widely-used approach. However, it comes with certain disadvantages: unrealistic assumptions (perfect divisibility and constant return to scale), arbitrarily-set threshold, and ignorance of opportunity cost. League table involves comparison of several health technologies simultaneously. The issue related to comparability between evaluation of each health technology limits its application. DM plane ensures improvement in health and sufficient resources mostly by disinvestment of currently funded programs. Its major disadvantages are difficulties in identification and disinvestment of such programs. Linear programming is, in theory, the best approach. Ideally, it requires sufficient data from all currently-funded or potential health technologies in conducting the analysis. Hence, the approach is somewhat impractical. Ongoing development in the field of data science and increasing availability of big data might enable its application in the near future. Given the mentioned shortcomings, pragmatic applications of league table and DM plane use them to evaluate competitive treatment programs for single health conditions. Using linear programming in prioritizing health programs was proven possible at the district level.
\end{abstract}

Keywords: Decision rule, Incremental cost-effectiveness ratio, Cost-utility analysis

\section{Introduction}

In countries with a Universal Healthcare Coverage (UHC) policy, such as Thailand, health systems are primarily financed by the public budget $[1,2]$. In Thailand, $80.1 \%$ of total health expenditure is from government revenue [3]. Sources of this funding typically consist of general government health expenditures and social health insurance [1]. This government intervention makes the healthcare market depart from a competitive market model. A major shortcoming is a lack of market-generated information about health technologies, including market prices. Therefore, economic evaluation (EE) of health technologies (including medicine, health intervention, and healthcare programs) plays an important role in the health system of countries with UHC. EE provides information for policymakers about health technologies. The information gained from EE could be used in making decisions regarding health technologies, such as whether or not to purchase technologies, how much should be paid for these technologies, etc.

There are 3 major types of EE in healthcare: cost-benefit analysis (CBA), cost-utility analysis (CUA), and cost-effectiveness analysis (CEA). Costs in all 3 types of EE are in monetary units. The difference between the 3 techniques is how the benefit from a health technology is assessed. In CBA, benefits from a health technology (e.g., improved health, future health spending saved, and quality of life) is converted into monetary units. In CEA, benefits from a health technology used in an analysis are 
http://wjst.wu.ac.th

common units related to corresponding health conditions (e.g., life year gained, number of new cases avoided, length of hospital stay), which are referred to as effectiveness. In CUA, benefits from health technology is converted into a generic unit, i.e., a single measure that incorporates both quantity and quality of life. The generic unit is referred to as utility. Examples of generic units include quality-adjusted life year (QALY), disability-adjusted life year (DALY), and healthy year equivalent (HYE). Because of difficulties in assigning monetary value to health outcomes, CBA is a less-applied technique in the EE of health technologies. Applications of CEA are also limited. CEA can only be used to compare health technologies for health conditions that have similar health outcomes. Using generic units, CUA is a more versatile technique compared to CEA. CUA is, hence, the most common form of EE in healthcare [4-6]. The focus of this review is on CUA.

CUA assesses a price paid (cost) for a health technology per a unit of utility (benefit gained) from that health technology. A cost per unit of utility is assessed in an incremental manner, comparing it with a referent scenario (i.e., do nothing or use another health technology). Hence, it is commonly referred to as an incremental cost-effectiveness ratio (ICER). Conventionally, to be considered cost-effective in CUA, a healthcare technology has to have its ICER be less than a particular threshold- namely, a threshold ICER. Therefore, this threshold ICER is a decision rule in CUA.

The concept of the threshold ICER as a decision rule in CUA was proposed by Weinstein and Zeckhauser [7]. Originally, a threshold ICER was proposed to be used in a setting where several health technologies are simultaneously assessed with a limited budget available to purchase those technologies. All relevant technologies are ranked by their ICERs in ascending order. The technologies are then selected for adoption from the first in the list (the one with the lowest ICER) until the budget is used up. The ICER of the last program selected, which uses up all the remaining budget, is a threshold ICER. This method of identifying a threshold ICER is called a 'league table' approach. In practice, however, individual, rather than all, relevant technologies are evaluated; hence, a threshold ICER is not identified from a league table. It is arbitrarily set. For example, the \$50,000-per-QALY threshold widely used in the US has been employed partly because it is a convenient figure [8]. Accordingly, the validity of using the threshold ICER as a decision rule has been questioned.

Arguments against the use of the threshold ICER includes ignorance of opportunity cost, unrealistic assumptions of constant return to scale and perfect divisibility, and its inconsistency with welfare economics [9-11]. Furthermore, the application of the threshold ICER has been suspected to cause a rise in healthcare expenditures, without ensuring maximized health benefits [12]. This paper reviews decision rules used in CUA and aims to identify better alternatives to the threshold ICER. The review explains the concepts, advantages, and disadvantages of each decision rule.

\section{Materials and methods}

Literature about decision rules for CEA/CUA were searched for using the following search terms: 'decision rule', 'economic evaluation', 'health technology assessment', 'cost-effectiveness analysis', 'cost-utility analysis', 'ICER', and 'incremental cost-effectiveness ratio'. The literature search was conducted on online databases via the Google Scholar website (https://scholar.google.co.th) and the PubMed website (http://www.ncbi.nlm.nih.gov/pubmed) for literature published up until July 2019. Relevant articles cited in articles identified from the literature search were followed and reviewed. The concepts, advantages, and disadvantages of each identified decision rules were extracted and summarized.

Guidelines for EE in healthcare from several countries, such as the UK, the US, and New Zealand, indicate QALY as a utility unit in CUA [8,13-16]. QALY is a measurement of health gain (life years) with adjustment for quality of life. One QALY is equal to 1 year in a perfect health state. Threshold ICERs mentioned in guidelines are in local currencies per QALY. 
http://wjst.wu.ac.th

\section{Results and discussion}

Four distinct decision rules, including the threshold ICER, were identified from the literature search. These included threshold ICER, league table, decision making plane, and linear programming. Some decision rules were referred to using different terminologies in different literatures. The concepts, advantages, and disadvantages of each decision rule are explained as follows.

\section{Threshold ICER}

\section{Concept}

In 1973, Weinstein and Zeckhauser proposed the use of a critical ratio in resource allocation [7]. Under a situation with limited resources, an efficient allocation of resources can be thought of as a constrained optimization problem. A solution to the optimization problem involves calculating a critical ratio, a benefit-cost ratio. Supposing that there are several projects waiting to be implemented, with a constraint of limited resources. It is impossible to execute all projects. Based on Weinstein and Zeckhauser's concept, benefit-cost ratios of all projects are first calculated. The project with the highest benefit-cost ratio is selected for implementation. Then, the project with the second highest benefit-cost ratio is selected. This process goes on until the available resources are used up. A benefit-cost ratio of the last project being implemented is called a critical ratio.

For resource allocation in the health sector, Weinstein and Stason [17] proposed the use of an inverse of the benefit-cost ratio, a ratio of cost to health gain from a health technology. A cost of resources used by the health technology is in a monetary unit. For CUA, health gain from the technology is expressed in generic units, among which QALY is the most widely used [4]. Health technologies are commonly assessed in comparison with the current practice (referent scenario). Both cost and health gain are expressed in an incremental manner $[4,17]$ as follows.

$$
I C E R=\frac{C_{i}-C_{0}}{E_{i}-E_{0}}
$$

where ICER $=$ incremental cost-effectiveness ratio

$$
\begin{aligned}
& C_{i}=\text { Cost of program } i \\
& C_{0}=\text { Cost of referent scenario } \\
& E_{i}=\text { Effectiveness of program } i \\
& E_{0}=\text { Effectiveness of benchmark scenario. }
\end{aligned}
$$

Lower ICER represents better value of a health technology (a unit of health gain (QALY) is purchased with a lower price). The threshold ICER indicates the payer's highest value of willingness and ability to pay for 1 QALY. A conventional decision rule is that a health technology with an ICER higher than the threshold ICER is not cost-effective and should not be funded.

\section{Advantages}

A decision to fund or not to fund a health technology based on a pre-defined threshold ICER is simple and transparent. This threshold ICER is employed by health policymakers in several countries. In the UK, the National Institute of Health and Care Excellence (NICE), a government agency responsible for health technology assessment, implicitly set 2 effective thresholds of $£ 20,000$ and $£ 30,000$. A health technology with ICER lower than $£ 20,000$ is usually classified as cost-effective; those with ICER between $£ 20,000$ and $£ 30,000$ are subject to additional considerations, such as degree of certainty, innovative nature of the technology, and impacts on non-health objectives. With an ICER higher than $£ 30,000$, the health technology is subject to more intensive considerations, having a higher likelihood of being classified as not cost-effective [18]. In Thailand, the Health Intervention and Technology Assessment Program (HITAP) plays the similar role to NICE in the UK [19]. The current threshold ICER used by HITAP is 1.0 - 1.2 times per-capita GDP per QALY, which is equal to $120,000-160,000$ Thai 
http://wjst.wu.ac.th

baht (THB) per QALY [20]. The World Health Organization (WHO) recommends the use of 3 times percapita GDP per 1 DALY averted as an appropriate threshold [21].

\section{Disadvantages}

There were arguments against using the threshold ICER as a decision rule. The arguments were that the concept of ICER relies on 2 strong assumptions: perfect divisibility and constant return to scale. These 2 assumptions were regarded as unrealistic by some authors $[9,11,12]$. To demonstrate the first point, suppose a new cancer treatment requires an investment in a state-of-the-art machine that has the capacity to treat 500 cases per year. Suppose the hospital has 100 cancer cases per year, one-fifth of the capacity. It is impossible for the hospital to purchase only a fraction of the whole machine in order to use only onefifth of its capacity. Hence, the assumption of perfect divisibility (ability to pay only the cost of each single unit of health improvement) is impractical.

For the second argument, constant return to scale implies constant marginal return cost for an additional unit of health technology, whereas economists usually assume diminishing marginal return (as reflected in the downward-slope demand curve) and increasing marginal cost (as reflected in the upwardslope supply curve). Therefore, ICER, which is basically marginal cost divided by marginal return, should increase with a number of units of health technology purchased rather than being constant. There is no reasonable explanation why healthcare should differ from other goods in this aspect.

Furthermore, when the use of a critical ratio in resource allocation was first introduced, the league table approach was used to identify the critical ratio. A league table consists of possible alternatives that potentially consume a limited pool of resources. Projects are chosen based on their benefit-cost ratios, which is an inverse of ICERs, until that pool of resources is exhausted. The cost of each project is considered at this step. The league table approach, hence, takes into account the opportunity cost of using resources.

Unlike the league table, the current application of threshold ICER is often conducted to evaluate an individual health technology against a referent scenario, not all possible alternative health technologies. The opportunity cost is the benefit forgone from the 'best' alternative use of the same amount of resource. Comparison only with a referent scenario, which is not necessarily the best alternative, does not strictly comply with the concept of opportunity cost in economics [10,22]. Moreover, threshold ICERs used in practice are not identified from the league table, as originally proposed by Weinstein and Zeckhauser, but are arbitrarily set. Therefore, the current practice of comparing an ICER with an arbitrarily-set threshold ignores the total cost of the health technology. It is also unclear how much the pool of resource left to be utilized is and where that pool of resource comes from. The total cost of health technology and the remaining budget is not taken into consideration in the current practice of using the threshold ICER as a decision rule. This practice obviously ignores opportunity cost.

Using the threshold ICER as a decision rule might entail a rise in healthcare expenditures $[9,11,12]$. When the threshold ICER is employed, a program with a positive lower-than-threshold ICER is likely to be funded. A positive ICER means that additional resources are required to fund the program, entailing a rise in expenditures. When it is not obvious where such resources come from, it is possible that the program partly consumes the resource used for other existing programs. If those existing programs are more cost-effective than the newly funded program, this process involves replacement of cost-effective programs by a less cost-effective one. Therefore, it is possible that the employment of threshold ICER could result in a simultaneous increase in healthcare expenditure and decrease in the total health benefits of the society.

In the US, the threshold of $\$ 50,000$ per QALY was widely cited as a decision rule for assessing cost-effectiveness. The $\$ 50,000$ threshold was the most popular value from 1990s in US literature and is still the most popular value used today [8]. This obviously shows that the threshold has not been adjusted for inflation over a long period of time. Grosse [23] tried to trace back the origin of this threshold. It turned out that the $\$ 50,000$ threshold was arbitrarily set, rather than based on 1980 s cost-effectiveness literature, as was widely understood. It became popular just because it was a convenient round number [23]. The $£ 20,000$ - 30,000 threshold used in the UK, and thresholds used in Ireland, suffer from a similar problem [24-26]. 
http://wjst.wu.ac.th

\section{League table}

Concept

The league table approach shares the same origins as the threshold ICER approach. The exact method proposed by Weinstein and Zeckhauser was employment of a league table of programs with benefit-cost ratio, total cost, and total benefit explicitly shown in the same league table [7]. A critical ratio (an inverse of threshold ICER) is actually a benefit-cost ratio of the last program on the league table that is being funded.

The application of league table in healthcare resource allocation was introduced by Weinstein and Stason [17]. The league table includes a range of health technologies ranked in ascending order of their ICER. As ICER represents an average cost per a QALY gained from a health technology, a lower ICER means a lower cost per unit of health benefit. In this approach, therefore, healthcare programs are chosen from the top of the table (a program with the lowest cost per QALY); decision makers go down the list until available resources are used up. The league table approach differs from the threshold ICER approach in 2 important aspects. First, the league table approach considers the cost-effectiveness of several potential health technologies at the same time. Secondly, the league table includes not only ICER, but also total cost and total benefit. A consideration of total cost against available budget is made explicit with this approach. It does not ignore opportunity cost as does the threshold ICER approach.

\section{Advantages}

As mentioned earlier, an advantage of the league table is that it takes into account the amount of resources (budget) available. This ensures that newly-funded programs have sufficient resources available for, and do not take resources from, existing programs. The other advantage is that it assesses several potential health technologies at the same time. Opportunity cost is considered in this approach.

\section{Disadvantages}

The last program in a league table being adopted relies on divisibility and constant return to scale assumptions, as does the threshold ICER approach. Healthcare programs typically do not follow the assumptions as discussed earlier. Another challenge in practice is that a process to identify which health technologies should be included in the league table takes time. After all alternatives are identified, waiting for complete information on all alternatives could further delay decision making. Moreover, if each program in a league table is evaluated independently, a choice of referent program used in the evaluation of each program will affect both cost and benefit, hence ICER. Differences in settings and methodologies also complicate comparison between programs in the league table. A protocol to assess validity and comparability of programs in a league able is required [27,28]. An alternative approach is to restrict application of the league table to evaluation of competitive programs for single health conditions. For instance, Ray et al. [29] conducted EE of 8 regimens for treating follicular non-Hodgkin's lymphoma.

\section{Decision making plane \\ Concept}

The decision making (DM) plane was introduced by Sendi et al. [30]. Its concept is that a currently funded program (program X) must be identified and replaced by a new program (program Y). For program $\mathrm{Y}$ to be considered cost-effective, the cancellation of program $\mathrm{X}$ must release sufficient resources to fund program $\mathrm{Y}$, and the benefits of program $\mathrm{Y}$ must be equal to or higher than program $\mathrm{X}$. This can be graphically presented in a so-called 'decision making plane' (Figure 1). 
http://wjst.wu.ac.th

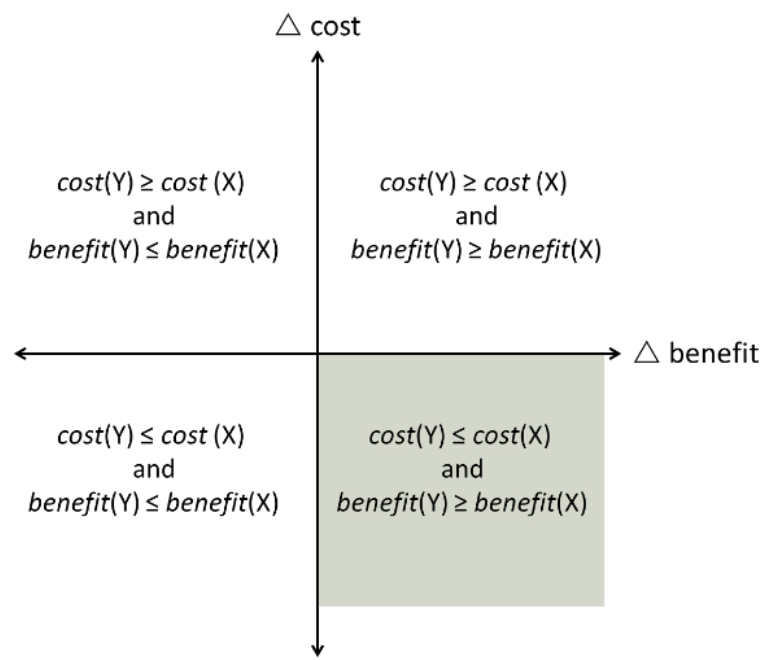

Figure 1 Decision making plane.

From Figure 1, cost-effectiveness criteria are fulfilled when the cost and benefits of program Y, in comparison with program $X$, falls into the southeast quadrant of the plane (the shaded area). In this quadrant, the cost of program $\mathrm{Y}$ is equal to or less than that of program $\mathrm{X}$ and the benefits of program $\mathrm{Y}$ are equal to or higher than those of program X. This involves an explicit comparison between benefits foregone from disinvesting program $\mathrm{X}$ and benefits gained from implementing program $\mathrm{Y}$. This is indeed a concept of opportunity cost.

The DM plane approach implicitly assumes no budget expansion in the healthcare sector. Under this condition, the advantages of DM plane include ensuring an increase in health and no reliance on divisibility and constant return to scale assumptions [31]. The threshold ICER, in contrast, cannot ensure a net health gain, and relies on those 2 assumptions.

\section{Advantages}

The advantages of this approach are that opportunity cost is explicitly considered and it does not rely on divisibility and constant return to scale assumptions. Moreover, this approach ensures that the new program produces at least an equal level of benefits compared to the replaced program; the total health benefits of the healthcare system increase or are, at least, unchanged. The situation in which this approach is most relevant is when new programs are yet to be funded without budget expansion. Under this scenario, replacement of existing programs is inevitable. This approach provides a framework for transparent consideration for adopting new programs.

\section{Disadvantages}

The identification of program $\mathrm{X}$ is difficult in the real world. Even if such a program is identified, it is not easy to cancel a healthcare program currently funded by the government (like program X) because of pressure from patient groups, as well as from physicians [32]. Nevertheless, disinvestment can occur in some circumstances. The real-world operation that is closest to the concept of DM plane is disinvestment in a drug when an alternative, providing better value for money, is available. This approach has been used by France's Transparency Commission and New Zealand's Pharmaceutical Management Agency (PHARMAC) [33]. 
http://wjst.wu.ac.th

\section{Linear programming}

\section{Concept}

Linear programming is a comprehensive solution for an optimization problem. In theory, it is possible to maximize health gain conditioning within a budget constraint, as well as within other constraints. Maximizing health gain from the limited available resources is indeed a definition of efficiency. In this approach, divisibility and constant return to scale assumptions are not required. The following shows a mathematical construct of this optimization problem.

$\operatorname{maximize} B=\sum_{i=1}^{n} b_{i}$

s.t. $\sum_{i=1}^{n} c_{i} \leq C$

where $B$ is total health benefits. $C$ is total resources available. $b_{i}$ is benefits from carrying out program $i$, and $c_{i}$ is resources consumed by program $i$. Total $n$ healthcare programs include currently-funded programs, as well as potential programs to be funded.

Unlike the threshold ICER approach, benefits and costs in this approach do not follow an incremental manner. In practice, this approach reviews all currently-funded programs, as well as potential programs to be adopted. Linear programming identifies the best combination of these programs in order to maximize the benefit to a system under resource constraints. This process is periodically undertaken, for instance, every fiscal year. All programs are subject to disinvestment in every round of revisions.

\section{Advantages}

In theory, linear programming is the most straightforward approach to achieve the efficiency objective [9]. This approach can incorporate all costs and constraints and provides the best combination of health technologies to be funded to maximize health gain.

\section{Disadvantages}

Obviously, information about the benefits and costs of all healthcare programs are required to carry out linear programming. As a large part of this required information has not yet been well-established, the applicability of this approach on a large scale is questioned [34]. Nevertheless, in the era of big data, increasing bodies of data in all sectors including healthcare, as well as an improvement in hardware and scientific methods to deal with big data, might enable this approach in the near future. There was a smallscale application of linear programming in prioritizing health interventions. A multi-criteria model to allocate human resources in community care programs (MARCCO), a model based on linear programming, was used to maximize health gain by prioritizing community care programs in 2 districts of Lisbon, Portugal [35].

\section{Conclusions}

Four distinct decision rules were identified from literature review, including the threshold ICER, league table, DM plane, and linear programming. All approaches have their advantages and disadvantages. In theory, linear programming is the best approach. However, lacking sufficient data to conduct the analysis limits its application. DM plane ensures improvement in health and sufficient resources. Its major disadvantages are difficulties in identification and disinvestment of currently funded programs. League table might be considered a more practical version of linear programming. However, it suffers similar disadvantages of linear programming, to a smaller degree, and shares some disadvantages of threshold ICER. The threshold ICER is, in practice, the most convenient approach. However, it is based on unrealistic assumptions, uses arbitrarily-set thresholds, and is ignorant of opportunity cost. 
http://wjst.wu.ac.th

\section{References}

[1] V Tangcharoensathien, W Patcharanarumol, Chitpranee, P Prakongsai, P Jongudomsuk, S Srithamrongsawat and J Thammathataree. Thailand health financing review 2010. SSRN Electron. J. 2010; 2010, 1-43.

[2] V Tangcharoensathien, W Patcharanarumol, P Ir, SM Aljunid, AG Mukti, K Akkhavong, E Banzon, DB Huong, H Thabrany and A Mills. Health-financing reforms in southeast Asia: Challenges in achieving universal coverage. Lancet $2011 ; 377,863-73$.

[3] The World Bank. Domestic general government health expenditure (\% of current health expenditure). The World Bank, 2019.

[4] MF Drummond, MJ Sculpher, GW Torrance, BJ O'Brien and GL Stoddart. Methods for the economic evaluation of health care programmes. Oxford University Press, 2005.

[5] PJ Neumann, GD Sanders, LB Russell, JE Siegel and TG Ganiats. Cost-effectiveness in health and medicine. Oxford University Press, 2016.

[6] LP Garrison, P Neumann, R Willke, A Basu, P Danzon, JA Doshi, MF Drummond, DN Lakdawalla, MV Pauly, CE Phelps, SD Ramsey, A Towse and MC Weinstein. A Health Economics Approach to US Value Assessment Frameworks-Summary and Recommendations of the ISPOR Special Task Force Report. Value Health 2018; 21, 161-5.

[7] M Weinstein and R Zeckhauser. Critical ratios and efficient allocation. J. Publ. Econ. 1973; 2,14757.

[8] P Neumann, JT Cohen and MC Weinstein. Updating cost-effectiveness: The curious resilience of the \$50,000-per-QALY threshold. New Eng. J. Med. 2014; 371, 796-7.

[9] S Birch and A Gafni. Cost effectiveness/utility analyses. Do current decision rules lead us to where we want to be? J. Health Eon. 1992; 11, 279-96.

[10] P Sendi, A Gafni and S Birch. Ethical economics and cost? Effectiveness analysis: Is it ethical to ignore opportunity costs? Expert Rev. Pharmacoecon. Outcomes Res. 2005; 5, 661-5.

[11] S Birch, A Gafni, O Ethgen and U Staginnus. Population needs, opportunity costs and economic methods for financial sustainability in health care systems. The Future of Health Economics. New York, USA, 2016, p. 191-202.

[12] A Gafni A and S Birch. Incremental cost-effectiveness ratios (ICERs): The silence of the lambda. Sos. Sci. Med. 2006; 62, 2091-100.

[13] PHARMAC. Guidelines for Funding Applications to PHARMAC. Amended in 2015. PHARMAC, 2015.

[14] PHARMAC. Cost-Utility Analysis (CUA) Explained. PHARMAC, 2015.

[15] B Santatiwongchai, V Chantarastapornchit, T Wilkinson, K Thiboonboon, W Rattanavipapong, DG Walker, K Chalkidou and Y Teerawattananon. Methodological variation in economic evaluations conducted in low-and middle-income countries: Information for reference case development. PloS One 2015; 10, e0123853.

[16] National Institute for Health and Care Excellence. Developing NICE guidelines: The manual. National Institute for Health and Care Excellence, 2018.

[17] MC Weinstein and WB Stason. Foundations of cost-effectiveness analysis for health and medical practices. New Eng. J. Med. 1977; 296, 716-21.

[18] J Earnshaw and G Lewis. NICE guide to the methods of technology appraisal. Pharmaco. Econom. 2008; 26, 725-7.

[19] A Mohara, S Youngkong, RP Velasco, P Werayingyong, K Pachanee, P Prakongsai, S Tantivess, $\mathrm{V}$ Tangcharoensathien, J Lertiendumrong, $\mathrm{P}$ Jongudomsuk and Y Teerawattananon. Using health technology assessment for informing coverage decisions in Thailand. Comp. Effect Res. 2012; 1, 137-46.

[20] Y Teerawattananon, N Tritasavit, N Suchonwanich and P Kingkaew. The use of economic evaluation for guiding the pharmaceutical reimbursement list in Thailand. Zeitschrift für Evidenz, Fortbildung und Qualität im Gesundheitswesen 2014; 108, 397-404. 
http://wjst.wu.ac.th

[21] World Health Organization. Investing in health for economic development. Report of the Commission on Macroeconomics and Health Geneva (Switzerland). World Health Organization, 2001.

[22] MY Bertram, Lauer JA, KD Joncheere, $\mathrm{T}$ Edejer, $\mathrm{R}$ Hutubessy, $\mathrm{MP}$ Kieny and $\mathrm{S}$ R Hill. Cost-effectiveness thresholds: Pros and cons. Bull. World Health Organ. 2016; 94, 925.

[23] SD Grosse. Assessing cost-effectiveness in healthcare: History of the $\$ 50,000$ per QALY threshold. Expert Rev. Pharmacoecon. Outcomes Res. 2008; 8, 165-78.

[24] C McCabe, K Claxton and AJ Culyer. The NICE cost-effectiveness threshold. Pharmaco. Econom. 2008; 26, 733-44.

[25] JF O'Mahony and D Coughlan. The Irish cost-effectiveness threshold: Does it support rational rationing or might it lead to unintended harm to Ireland's health system? Pharmaco. Econom. 2016; 34, 5-11.

[26] National Institute for Health Care Excellence. Developing NICE guidelines: The manual. National Institute for Health and Care Excellence, 2015.

[27] K Gerard and G Mooney. QALY league tables: Handle with care. Health Econ. 1993; 2, 59-64.

[28] M Drummond, G Torrance and J Mason. Cost-effectiveness league tables: More harm than good? Soc. Sci. Med. 1993; 37, 33-40.

[29] JA Ray, E Carr, G Lewi and R Marcus. An evaluation of the cost-effectiveness of rituximab in combination with chemotherapy for the first-line treatment of follicular non-Hodgkin's lymphoma in the UK. Value Health 2010; 13, 346-57.

[30] P Sendi, A Gafni, and S Birch. Opportunity costs and uncertainty in the economic evaluation of health care interventions. Health Econ. 2002; 11, 23-31.

[31] S Birch and A Gafni. Population Needs, Opportunity Costs and Economic Methods for Financial Sustainability in Health Care Systems. The Future of Health Economics, 2016, p. 191-202.

[32] C Harris, K Allen, W Ramsey, R King and S Green. Sustainability in Health care by Allocating Resources Effectively (SHARE) 11: Reporting outcomes of an evidence-driven approach to disinvestment in a local healthcare setting. BMC Health Serv. Res. 2018; 18, 386.

[33] B Parkinson, C Sermet, F Clement, S Crausaz, B Godman, S Garner, M Choudhury, SA Pearson, R Viney, R Lopert and AG Elshaug. Disinvestment and value-based purchasing strategies for pharmaceuticals: An international review. Pharmaco. Econom. 2015; 33, 905-924.

[34] S Simoens. How to assess the value of medicines? Front. Pharmacol. 2010; 1, 115.

[35] MD Oliveira, TC Rodrigues, CAB Costa and ABD Sá. Prioritizing health care interventions: A multicriteria resource allocation model to inform the choice of community care programmes. Advanced decision making methods applied to health care. Springer, 2012, p. 141-54. 